\title{
Mise en evidence de substances fluorescentes dans les organes jaunes de Caecosphaeroma burgundum Dollfus, Crustacé Isopode des eaux souterraines
}

\author{
Par H. Descimon ${ }^{1}$ ) et Cl. Marvillet ${ }^{2}$ )
}

Avec 2 figures dans le texte

Le Crustacé Caecosphaeroma burgundum, observé à l'état vivant, montre des taches jaunes réparties assez régulièrement sur tout le corps, mais particulièrement développées dans le prosoma et le pléotelson (Fig. 1). J. Daum, qui a étudié le premier la biologie de ce Sphéromien cavernicole dans le laboratoire du Professeur Husson, les a décrits sous le nom d'«organes jaunes» (R. Husson et J. Daum 1953; J. Daum 1954).
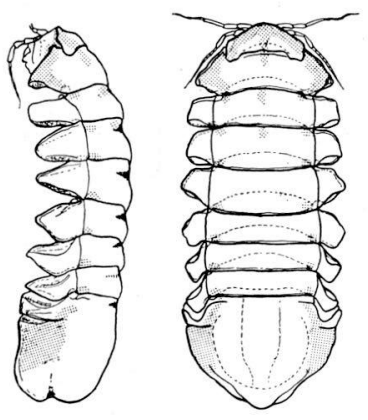

Fig. 1. Répartition des organes jaunes (en pointillé) chez Caecosphaeroma burgundum Dollfus examiné en position latérale (à gauche) et en face dorsale (à droite). On remarque la forte concentration de ces organes dans la partie antérieure du corps (tête et premier segment thoracique) et au niveau du pléotelson. Ces organes jaunes sont situés juste sous la carapace. (D'après J. Daum, 1954.)

Homologues des organes de Zenker, décrits dans la partie postérieure du corps d'Asellus aquaticus L., ils ont été retrouvés (R. Husson et J. Daum 1957) chez l'autre espèce du genre Caecosphaeroma (C. virei Dollfus des grottes du Jura) et dans deux autres Isopodes du genre Asellus, l'un épigé, A.meridianus Racovitza, l'autre hypogé, A.cavaticus Leydig (R. Husson et J. P. Henry 1963).

1) Laboratoire de Zoologie, Ecole Normale Supérieure - 24 rue Lhomond, Paris-Vo.

$\left.{ }^{2}\right)$ Laboratoire de Biologie Animale et Générale-Faculté des Sciences 21, Dijon. 
D'après J. Daum, il s'agit de conglomérats de cellules adipeuses de couleur jaune, contenant de nombreuses sphérules de diamètres variés, concrétions d'acide urique ou d'urates.

Les recherches entreprises par H. Schorr dans le même laboratoire, en utilisant les méthodes microchimiques mises au point par J. Schindler en 1950, ont mis en évidence la présence d'acide urique dans les concrétions des organes jaunes et l'absence d'urates et de guanine, sans pouvoir affirmer la présence éventuelle d'autres purines (R. Husson et H. Schorr 1957; H. Schorr 1957). La localisation de l'acide urique au sein même des cellules, attribuée aux sphérules, n'y était cependant pas déterminée de façon exacte.

Quant au pigment jaune responsable de la coloration, il entrelace tout l'organe d'un fin réseau irrégulier, indépendant des sphérules. La recherche des caroténoïdes par méthodes microchimiques, faite par Schorr, était demeurée négative.

Nous avons repris méthodiquement l'étude de ces pigments jaunes de façon à tenter la détermination de leur nature exacte.

\section{Vérifications préliminaires}

L'observation, au microscope polarisant, d'organes jaunes dilacérés, confirme la structure cristalline des abondantes sphérules qui représentent très probablement l'acide urique au sein des cellules.

Des chromatogrammes ont été effectués sur couche mince de silicagel à l'aide d'extraits d'organes jaunes et de spots témoins de carotènes. Leur révélation, par vaporisation de trichlorure d'antimoine en solution dans le chloroforme anhydre, reste négative et confirme l'absence de caroténoïdes dans les organes jaunes.

\section{Matériel et techniques}

Les animaux ont été récoltés dans le cours d'eau souterrain de l'abîme de Bévy (Côte-d'Or) et mis en élevage dans les chambres froides du laboratoire.

La technique analytique employée fait appel essentiellement à la chromatographie sur papier et couche mince de cellulose et à l'électrophorèse sur couche mince de cellulose.

Les animaux (110 individus, soit 3,5 g) sont broyés dans un petit broyeur de Potter, dans l'éthanol-chloroforme (1:1) (c.a. $5 \mathrm{ml} / \mathrm{g}$ ). Le broyat est centrifugé à $1000 \mathrm{~g}$, le surnageant est éliminé et l'opération est répétée dans les mêmes conditions. 
Le culot est repris dans $2,5 \mathrm{ml} / \mathrm{g}$ de méthanol-pyridine-eau (5:1:4), additionné de thiodiglycol $(0,5 \%)$, puis centrifugé; l'extraction est recommencée une seconde fois et les extraits sont fusionnés.

Pour les études individuelles, les animaux, ou les organes disséqués, sont simplement broyés une fois dans le méthanol-pyridine-eau et centrifugés.

Les chromatographies bidimensionnelles sont effectuées en série sur des feuilles $20 \times 20$ de papier Whatman no 1 . La combinaison de solvants utilisée est $n$-propanol-ammoniaque $1 \%(2: 1)$ puis $n$-butanolacide acétique-eau (20:3:7).

Quelques chromatographies unidimensionnelles ont été réalisées de la même manière dans le propanol-ammoniaque pour comparer divers organes dont les extraits ont été chromatographiés côte à côte.

L'extrait est évaporé à l'aide d'une microseringue à l'origine d'un chromatogramme (volume déposé : $50 \mu \mathrm{l}$ pour une chromatographie bidimensionnelle, $25 \mu \mathrm{l}$ pour une chromatographie unidimensionnelle).

D'autre part, des chromatographies semi-préparatives ont été effectuées sur papier Whatman 3 MM $20 \times 30 \mathrm{~cm}$ préalablement lavé; $1,5 \mathrm{ml}$ d'extrait est évaporé en ligne selon la petite dimension. Le solvant de chromatographie est le n-propanol-ammoniaque. Les bandes fluorescentes et absorbantes obtenues sont éluées dans l'eau (additionnée de $0,5 \%$ de thiodiglycol); l'éluat est ensuite lyophilisé et repris dans le méthanol-pyridine et utilisé pour la chromatographie unidimensionnelle en comparaison avec des substances témoins dans divers solvants.

L'électrophorèse est pratiquée sur couche mince de cellulose (tampon : acétate d'ammonium $\mathrm{pH} 6,3 ; 20 \mathrm{~V} / \mathrm{cm}$ ).

Les chromatogrammes et électrophorèse sont lus en lumière U.V. (365 et $254 \mathrm{~m} \mu$ ). Les substances puriques sont révélées par immersion dans l'éosine mercurique $\left(0,2 \%\right.$ d'éosine dans l'éthanol à $95^{\circ}$ saturé de chlorure mercurique), puis lavage dans l'alcool à $95^{\circ}$; les chromatogrammes sont observés en lumière U.V. Cette technique, très sensible, sera détaillée dans une publication ultérieure.

\section{Résultats}

\section{A. Etude des substances fluorescentes}

Six taches fluorescentes, dont certaines, bleues, apparaissent rapidement sous irradiation aux U.V., et deux taches absorbantes peuvent être observées sur les chromatogrammes bidimensionnels (Fig. 2). 
Parmi elles, les plus abondantes, 3 (violette), 6 (jaune) et 4 (absorbante) ont été plus particulièrement étudiées.

$1^{0}$ - Tache $n^{\circ} 3$. La bande correspondant à cette tache sur les chromatogrammes semi-préparatifs a été comparée avec l'isoxanthoptérine qui présente la même couleur de fluorescence et des valeurs de RF similaires. Une comparaison entre la substance 3 et l'isoxanthoptérine de synthèse a été effectuée dans 5 solvants choisis parmi ceux qui donnent la meilleure différenciation (Tabl. 1). Les RF coïncident dans tous les cas, une légère différence étant cependant

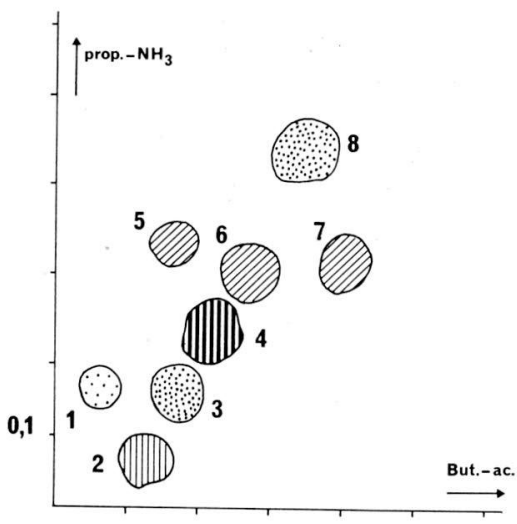
observée dans les chlorure d'ammonium. Les migrations électrophorétiques sont également identiques (déplacement de 0,45-0,60 cm en 2 heures vers la cathode).

Fig. 2. Chromatogramme bidimensionnel d'extrait de Caecosphaeroma burgundum : substances fluorescentes et absorbantes dans l'U.V.1: bleu. 2 : absorbant. 3 : violet. 4 : absorbant. $5,6,7$ : jaune ou jaune-vert. 8 : violet.

$2^{\circ}$ - Tache $n^{\circ} 6$. Cette substance a été comparée avec de la riboflavine et de la sépiaptérine témoin extraite du Papillon Colias croceus Fourcroy (H. Descimon 1965). Les valeurs de RF permettent d'exclure l'hypothèse que cette substance soit de la sépiaptérine; de même, alors que ce dernier composé s'altère rapidement sur les chromatogrammes en acide ptérine-6-carboxylique (la couleur de fluorescence passant progressivement du jaune au bleu), le pigment jaune de Caecosphaeroma reste stable dans les mêmes conditions. Ce fait permet d'écarter également la "xanthoptérine $\mathrm{B}_{2}$ " (M. Goto, M. Konishi, K. Sugiura, M. Tsusue 1966), elle aussi très labile. En revanche, cette stabilité relative rapproche la substance 6 de la riboflavine; les valeurs des RF ne présentent aussi que des différences légères, qui peuvent n'être dues qu'à l'impureté du produit chromatographié. Une étude sur de plus grandes quantités s'avère done nécessaire.

$3^{\circ}-$ Tache no 4. Celle-ci s'est révélée identique, par ses propriétés chromatographiques (Tabl. 1) et par sa révélation à l'éosine mercurique, à de l'acide urique synthétique. Ce résultat confirme les conclusions de H. Schorr. 


\section{Tableau 1}

Valeur des RF dans les différents solvants utilisés. PA: n-propanol-ammoniaque $1 \%(2: 1)$. BA: n-butanol-acide acétique-eau (20:3:7). IAA: isopropanol-acétate d'ammonium $2 \%(1: 1)$. $\mathrm{NH}_{4} \mathrm{Cl}: 3 \%$. PEE : pyridineéther-eau (4:3:3). FP : formiate de pyridine $\mathrm{pH} 6,5-0,1 \mathrm{~N}$. BPE : n-butanolpyridine-eau $(6: 4: 3)$

\begin{tabular}{lccccccc}
\hline & $\begin{array}{c}\text { Tache } 3 \\
\text { violet }\end{array}$ & $\begin{array}{c}\text { Isoxantho- Tache 6 } \\
\text { ptérine } \\
\text { témoin }\end{array}$ & $\begin{array}{c}\text { Sépia- } \\
\text { jaune } \\
\text { vert }\end{array}$ & $\begin{array}{c}\text { Ribo- } \\
\text { Colias }\end{array}$ & $\begin{array}{c}\text { Tache 4 } \\
\text { flavine } \\
\text { absor- } \\
\text { bante }\end{array}$ & $\begin{array}{c}\text { Acide } \\
\text { urique }\end{array}$ \\
\hline $\mathrm{PA}$ & 0,16 & 0,17 & 0,30 & 0,32 & 0,32 & 0,22 & 0,22 \\
$\mathrm{BA}$ & 0,19 & 0,20 & 0,27 & 0,34 & 0,29 & 0,19 & 0,19 \\
$\mathrm{IAA}$ & 0,39 & 0,38 & 0,40 & 0,49 & 0,42 & & \\
$\mathrm{NH}_{4} \mathrm{Cl}$ & 0,30 & 0,33 & 0,31 & 0,30 & 0,35 & & \\
$\mathrm{PEE}$ & 0,62 & 0,62 & 0,77 & 0,74 & 0,79 & & \\
$\mathrm{FP}$ & & & & & & 0,50 & 0,50 \\
$\mathrm{BPE}$ & & & & & & 0,13 & 0,13
\end{tabular}

La substance 2, absorbante, est probablement une purine, puisqu'elle réagit fortement à l'éosine mercurique. La comparaison avec des substances de synthèse (guanine, adénine, xanthine et hypoxanthine) n'a permis de l'identifier à aucun de ces composés.

La substance 8, présente en trop faible quantité, n'a pu être soumise à des essais systématiques, mais il est possible qu'il s'agisse de bioptérine.

L'appararition, sous irradiation U.V., de taches fluorescentes bleues, laisse à penser que des tétrahydroptérines sont présentes chez cet animal (I. Ziegler 1965; M. Goto, M. Konishi, K. Sugiura, M. Tsusue 1966).

\section{B. Répartition des substances fluorescentes selon les organes}

Des chromatogrammes unidimensionnels comparatifs d'extraits de différents organes (pléotelson, hépatopancréas, carapace et tête) montrent des différences notables dans les teneurs en substances fluorescentes ou absorbantes (Tabl. 2).

Tableau 2

Répartition des substances fluorescentes et absorbantes suivant les organes

\begin{tabular}{lccccc}
\hline & 2 & 3 & 4 & 6 & 8 \\
\hline $\begin{array}{l}\text { Pléotelson } \\
\text { Hépatopancréas }\end{array}$ & +++ & ++ & ++ & ++ & \\
$\begin{array}{l}\text { Carapace } \\
\text { Tête }\end{array}$ & & & + & & + \\
& & ++ & ++ & ++ &
\end{tabular}


L'examen de ce tableau montre que la substance 6 est particulièrement abondante dans les régions où sont localisés les organes jaunes. Cette substance est donc très probablement le pigment jaune responsable de leur vive coloration.

La substance 3 est, elle aussi, particulièrement abondante dans ces organes, de même que l'acide urique.

Une comparaison entre des extraits d'animaux laissés à l'obscurité et d'autres placés à la lumière ambiante (1000 lux environ) n'a pas montré de différence notable.

\section{Discussion}

Les seuls travaux sur les pigments et substances fluorescentes des Crustacés n’ont porté que sur les Décapodes (R. G. Busnel et A. Drilhon 1948 ; M. Viscontini, H. Schmidt et E. Hadorn 1955). Aucun travail de ce genre n'a été effectué sur les pigments fluorescents des Isopodes et, a fortiori, sur ceux des Isopodes cavernicoles. La présente étude montre l'existence d'un certain nombre de ces substances. La substance 3 est vraisemblablement de l'isoxanthoptérine, composé extrêmement répandu chez la plupart des Arthropodes. Le pigment jaune (substance 6) n'a pu être identifié avec certitude. Des séparations à l'échelle préparative seront effectuées ultérieurement pour déterminer sa structure. Des travaux récents ont d'ailleurs montré la variété des pigments jaunes des Arthropodes (M. Viscontini 1963; G. H. Schmidt et M. Viscontini 1964; H. Descimon 1965 ; M. Goto et al. 1966). La présence éventuelle de tétrahydroptérines est également assez vraisemblable.

Dans le domaine de la faune cavernicole, R. Bernasconi (1964) a obtenu d'intéressants résultats concernant les Coléoptères troglobies, chez lesquels il a pu mettre en évidence la présence de leucoptérine et de xanthoptérine.

Le rôle physiologique de ces pigments reste à élucider; des recherches récents ont montré la grande importance comme coenzymes d'oxydation des substances possédant le noyau ptéridine et en particulier des pigments jaunes (M. Viscontini et G. H. Schmidt 1965). La biologie très particulière de Caecosphaeroma burgundum est peut-être en relation avec l'accumulation frappante d'une telle substance chez cet animal.

\section{RÉSUMÉ}

L'analyse chromatographique des organes jaunes du Crustacé Isopode des eaux souterraines Caecosphaeroma burgundum Dollfus a permis de mettre en évidence, par observation des chromatogrammes en lumière ultra-violette, un certain nombre de substances absorbantes et de substances fluorescentes. Parmi ces dernières, seule l'isoxanthoptérine a été identifiée. 
Le pigment jaune, qui possède vraisemblablement le noyau ptéridine, n’a pu être identifié à un composé connu et fera l'objet de travaux ultérieurs.

\section{SUMMARY}

Chromatographic analysis of the yellow organs of the fresh-water Isopod Crustacean Caecosphaeroma burgundum Dollfus revealed, through observation of the chromatograms in ultraviolet light, the occurence of both absorbant and fluorescent substances. Among the latter, only isoxanthopterin has been identified. The yellow pigment, which probably has a pteridine nucleus, could not be identified as a known compound and will be the object of later investigations.

\section{B I B LI O GRAP H I E}

Bernasconi, R. (1964) - Recherches biochimiques sur les pigments des Insectes cavernicoles. Ann. de Spéléol., 19, 3: 553-572.

Busnel, R. G., et Drilhon, A. (1948) - Sur les pigments flaviniques et ptériniques des Crustacés. Bull. Soc. Zool. Fr., 73: 141-185.

DA u, J. (1954) - Zur Biologie einer Isopodenart unterirdischer Gewässer: Caecosphaeroma burgundum Dollfus. Ann. Univ. Sarav., 3: 104-160.

Descimon, H. (1965) - Identification de la sépiaptérine dans les ailes des Pieridae (Lepid. Rhopalocera). Bull. Soc. Chim. Biol., 47, $\mathrm{n}^{0}$ 6: 1095-1100.

Goto, M., Konishi, M., Sugiura, K., et Tsusue, M. (1966) - The structure of a yellow pigment from the mutant lemon of Bombyx mori. Bull. Chem. Soc. Jap., 39, no 5 .

Husson, R., et Daum, J. (1953) - Sur la Biologie de Caecosphaeroma burgundum. C.R. Ac. Sc., $236: 2345-2347$.

- - (1957) - Répartition des organes jaunes chez le troglobie Caecosphaeroma burgundum Doll. Notes biospéol., 12: 53-57.

Husson, R., et Henry, J. P. (1963) - Etude chez trois espèces du genre Asellus des concrétions des organes de Zenker. Bull. Soc. Zool. Fr., $88: 274-277$.

Husson, R., et Schorr, H. (1957) - Mise en évidence d'acide urique dans les organes jaunes du Crustacé troglobie Caecosphaeroma burgundum Dollfus. C.R. Ac. Sc., 244: 513-515.

Schindler, J. (1950) - Reservestoff- und Exkretspeicherung bei Bodentieren unter besonderer Berücksichtigung der Harnsäureverbindungen. Österr. Zool. Zeitschr. 2: 517-567.

Schmid t, G. H., et Viscontini, M. (1963) - Fluoreszierende Stoffe aus roten Waldameisen der Gattung Formica. Isolierung einer RiboflavinFormicapterin-Verbindung. Helvet. Chim. Acta, 47, fasc. 7.

Schorr, H. (1957) - Untersuchungen über Purine und Pigmente in den ,Gelben Organen" des Höhlenisopoden Caecosphaeroma burgundum Doll. Ann. Univer. Sarav.-Naturwiss., V: 311-328.

Viscontini, M. (1963) - Ptérines et génétique chez Drosophila melanogaster. Il Farmaco, no 1 .

Viscontini, M., et Sсниid t, G. H. (1965) - Über die physiologische Bedeutung der Pterine. Zeitschr. für Naturforsch., 20 b: 327-331.

Viscontini, M., Schmidt, H., et Hadorn, E. (1955) - Isolierung fluoreszierender Stoffe aus Astacus fluviatilis. Exper., XI : 390-392.

Ziegler, I. (1965) - Pterine als Wirkstoffe und Pigmente. Ergeb. Physiol., biol. Chem. und exper. Pharmak., 56. 